\title{
Experimenting with robotic intra-logistics domains
}

\author{
MARTIN GEBSER, PHILIPP OBERMEIER, THOMAS OTTO \\ and TORSTEN SCHAUB \\ University of Potsdam, Germany \\ ORKUNT SABUNCU \\ TED University, Ankara, Turkey \\ VAN NGUYEN and TRAN CAO SON \\ New Mexico State University, Las Cruces, USA \\ submitted 30 April 2018; accepted 11 May 2018
}

\begin{abstract}
We introduce the asprilo $^{1}$ framework to facilitate experimental studies of approaches addressing complex dynamic applications. For this purpose, we have chosen the domain of robotic intralogistics. This domain is not only highly relevant in the context of today's fourth industrial revolution but it moreover combines a multitude of challenging issues within a single uniform framework. This includes multi-agent planning, reasoning about action, change, resources, strategies, etc. In return, asprilo allows users to study alternative solutions as regards effectiveness and scalability. Although asprilo relies on Answer Set Programming and Python, it is readily usable by any system complying with its fact-oriented interface format. This makes it attractive for benchmarking and teaching well beyond logic programming. More precisely, asprilo consists of a versatile benchmark generator, solution checker and visualizer as well as a bunch of reference encodings featuring various ASP techniques. Importantly, the visualizer's animation capabilities are indispensable for complex scenarios like intra-logistics in order to inspect valid as well as invalid solution candidates. Also, it allows for graphically editing benchmark layouts that can be used as a basis for generating benchmark suites.
\end{abstract}

\section{Introduction}

Answer Set Programming (ASP; Lifschitz 1999) has come a long way, starting as a semantics for logic programming, over having increasingly performant systems, to a growing number of significant applications in academia and industry. However, this development is threatened by a lack of complex benchmark scenarios mimicking the needs of real-world applications. In contrast to many available benchmark suites, often supplied by automatic instance generators, real-world applications are rarely disseminated, either because they are classified or come only with a handful of instances. Another commonality of existing benchmark suites is that they are kept simple, stick to basic ASP, and usually feature at most a single specifics, so that they can be processed by as many systems as possible. However, this is in contrast to many real-world applications whose solution requires the integration of multiple types of knowledge and forms of reasoning. Last but

1 asprilo stands for Answer Set Programming for robotic intra-logistics. 
not least, a feature distinguishing ASP from all other solving paradigms is its versatility, which is best put in perspective by solving multi-faceted problems.

We fear that the lack of complex benchmark scenarios becomes a major bottleneck in ASP's progression towards real-world applications, and hence that more and more should be made available to our community. As a first step to overcome this problem, we have identified the domain of robotic intra-logistics, a key domain in the context of the fourth industrial revolution, as witnessed by Amazon's Kiva, GreyOrange's Butler, and Swisslog's CarryPick systems. ${ }^{2}$ All of them aim at automatizing warehouse operations by using robot vehicles that drive underneath mobile shelves and deliver them to picking stations. From there, workers pick and place the requested items in shipping boxes. Apart from the great significance of this real-world domain, our choice is motivated by several aspects. First of all, the warehouse layout is grid-based and thus provides a suitable abstraction for modeling robot movements in ASP. Moreover, the domain offers a great variety of manifold problem scenarios that can be put together in an increasingly complex way. For instance, one may start with single or multi-robot pathfinding scenarios induced by a set of orders that are accomplished by using robots for transporting shelves to picking stations. This can be extended in various ways, for example, by adding shelf handling and delivery actions, considering order lines with multiple product items, keeping track of the number of ordered and/or stored product items, modeling energy consumption and charging actions, taking into account order frequencies, supplies, and priorities, striving for effective layouts featuring dedicated locations, like highways or storage areas, and so on. Finally, the domain is extremely well-suited for producing scalable benchmarks by varying layouts, robots, shelves, orders, product items, etc. Inspired by this, we have developed the benchmark environment asprilo consisting of four parts (i) a benchmark generator, (ii) a solution checker, (iii) a benchmark and solution visualizer, and (iv) a variety of reference encodings. The design of asprilo was driven by the desire to create an easily configurable and extensible framework that allows for generating scalable, standardized benchmark suites that can be visualized with and without a corresponding solution. Correctness can be established via a modular solution checker. The accompanying reference encodings may serve as exemplary bases for extended encodings addressing more complex scenarios. We use them in Section 5 to illustrate asprilo's usage and value for benchmarking. In particular, we show how asprilo may allow us to understand the virtues of different ASP techniques. The asprilo framework is freely available at https://potassco.org/asprilo.

\section{2 asprilo's benchmark generation component}

\subsection{Problem domains}

All asprilo domains aim at capturing abstractions of intra-logistics systems involving multiple robots, shelves, and stations, placed in a warehouse environment, along with a set of orders. We begin with a description of the common setting.

Given a set of orders, the idea is to have robots bring shelves containing requested products to picking stations until all orders are satisfied. The warehouse floor is laid

2 www.amazonrobotics.com, www.greyorange.com/products/butler, www.swisslog.com/ carrypick 
out as a two-dimensional grid of squares. A square can be declared as highway, picking station, ${ }^{3}$ or storage location. Only the latter can be occupied by shelves without being carried by robots. A shelf stores products in certain quantities, and a picking station accepts product units requested by orders. Both occupy a single grid square. A square may be occupied by at most one robot and one shelf, no matter whether the robot carries the shelf or not. Hence, robots may pass or park underneath shelves, unless they carry one. Highways are used to declare transit areas for shelf carrying robots; they are neither shelf storage locations nor parking lots for idle robots. ${ }^{4}$

Per time step, a robot can perform at most one action, either ${ }^{5}$ move to an adjacent square, pick up or put down a shelf, or deliver (products on) a shelf to a picking station. Robots must not swap squares at a time step. A robot may pick up a shelf, provided that both are on the same square and it does not yet carry a shelf; it may put down a shelf, if it carries the shelf. Thus, a robot can pick up a shelf, carry it while moving, and put it down eventually. However, a robot may never put down a shelf outside the storage area. A robot carrying a shelf may deliver product units from the shelf at a picking station, ${ }^{6}$ provided it occupies the corresponding square and the product units are part of an order line processed at this station. The specific arrangement of deliveries distinguishes several asprilo domains and is made precise below.

An order is a non-empty set of order lines, which are requests for products in certain quantities to be delivered to a given picking station. For simplicity, ${ }^{7}$ all requested products are available in sufficient quantity on the shelves in the warehouse. An order line is fulfilled if the requested amount of product units has been delivered to the designated picking station. An order is fulfilled if all its order lines are fulfilled. In the current setting, all orders are provided initially (instead of arriving over time). In these terms, the overall goal can be rephrased to find a parallel plan of robot actions that fulfills all orders.

In what follows, we introduce selected problem domains of asprilo. We begin with the (currently) most general setting and gradually refine it afterwards to obtain simplified variants. In the general asprilo domain $\mathbf{A}$, deliveries fully account for the quantities of product units taken off a shelf. A delivery action deals with a single order line; the number of transferred units can neither exceed the quantity available on the shelf nor the requested amount of units. Thus, multiple order lines require multiple deliveries, and one order line may require units from several shelves.

The asprilo domain $\mathbf{B}$ simplifies A by ignoring product quantities. A delivery action still deals with a single order line but only the requested product must be on the shelf (and remains after delivery), no matter how many units are available or requested. The asprilo domain $\mathbf{C}$ further simplifies $\mathbf{A}$ and $\mathbf{B}$ by making one delivery action deal at once with all pending order lines matching products on the shelf. Thus, in this setting, one deliver action may fulfill several orders at a picking station all at once.

The asprilo domain $\mathbf{M}$ is a drastic simplification designed to resemble multi-agent path finding scenarios known from the literature (cf. Section 6). This domain features only move actions and the only concern is to find a parallel plan that positions each robot

3 Other dedicated stations, like charging or supplying stations, are foreseen in future editions of asprilo.

4 We are currently not distinguishing parking areas for robots and use storage locations for simplicity.

5 More actions, like battery charging or restocking, are planned but not yet part of asprilo.

6 In reality, this is usually a passive action since a person or a robot picks items from the shelf.

7 Supply management is another possible future extension of asprilo. 
under a specific shelf. Hence, shelves remain on the spot and no products are delivered. To accommodate this scenario in the asprilo framework, we begin by equipping each shelf with a unique product. The number of robots and orders is aligned, and each order lines up a unique product. All ordered products are stocked, at most one per shelf. An order (line) is fulfilled if some robot is located under the shelf holding a requested product. Note that robots are not pre-assigned to shelves in $\mathbf{M}$, as common in certain multi-agent path finding scenarios. The addition of such an assignment constitutes yet another variant, and corresponds to the traditional MAPF problem, as detailed in Section 6.

To bridge the gap between scenarios $\mathbf{A}, \mathbf{B}, \mathbf{C}$ and $\mathbf{M}$, we also consider the restriction of the former to the specific shelf and order alignment used in the latter. The resulting scenarios $\mathbf{A}^{\mathbf{M}}, \mathbf{B}^{\mathbf{M}}$, and $\mathbf{C}^{\mathbf{M}}$ thus deal with full-fledged delivery scenarios but only singleton orders and shelves with unique product units. This allows us to experiment with the general functionality of $\mathbf{A}, \mathbf{B}$, and $\mathbf{C}$ in the rather restricted warehouse setting of $\mathbf{M}$.

Clearly further constraints can be imposed, leading to a wide variety of asprilo scenarios.

\subsection{Instance format}

Our instance format follows the well-known object-attribute-value scheme that provides a generic and extensible knowledge representation format. More precisely, we represent each initial item as a fact of binary predicate init/2 of form

init (object $(T, I)$, value $(A, V)$ ).

where $T$ is an object type, $I$ a (relative) object identifier, $A$ an attribute, and $V$ its value. The current object types along with their admissible attributes are as follows:

$\begin{array}{ll}\text { node } & \text { at } / 2 \\ \text { highway } & \text { at } / 2 \\ \text { robot } & \text { at } / 2 \text {, carries/1 } \\ \text { shelf } & \text { at } / 2 \\ \text { pickingStation } & \text { at } / 2 \\ \text { product } & \text { on } / 2 \\ \text { order } & \text { line/2, pickingStation/1 }\end{array}$

The trailing number indicates the number of arguments of each attribute. For instance, the fact 'init (object (robot, 34), value (at, $(2,3))$ ).' represents that robot 34 is at position $(2,3)$. Note that all but the last type in the above list are part of the initial layout of a warehouse and its inventory. Each node has a unique identifier as well as a two-dimensional position. Optionally, a position can be declared as a highway. The major entities, namely, robots, shelves, and picking stations, have (initial) positions. Also, a robot may carry a shelf. The type product gives the inventory by listing how many product items are stored on a shelf. Finally, each order consists of one or more order lines, each fixing how many product items are requested, and its destination, namely, the picking station where the order is put together.

\subsection{Instance generator}

The instance generator of asprilo allows for generating benchmark sets with variable features and degrees of difficulty. Each instance is expressed in the format described 


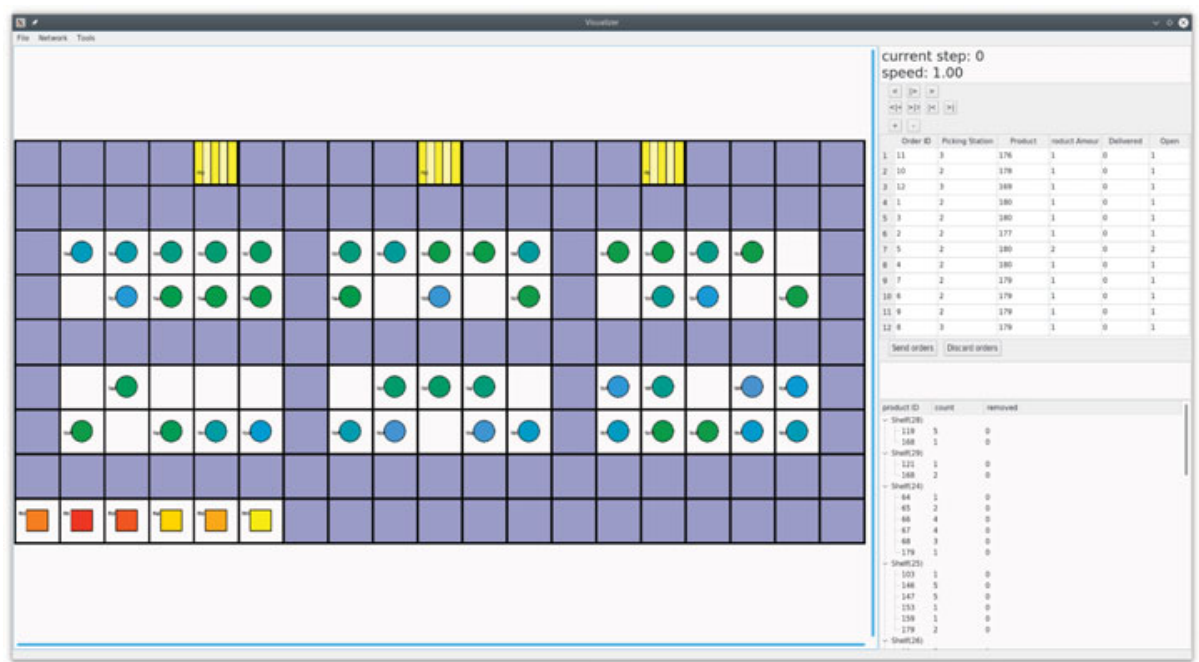

Fig. 1. Exemplary asprilo screenshot: The main window gives a warehouse layout; no plan is loaded. Picking stations are represented by striped yellow squares, shelves by solid circles, and robots by solid squares. Highways are brought out in purple. The side windows provide controls for plan animation, and give details about the current orders and the warehouse inventory.

above; its name is standardized to reflect its major characteristics, viz. the grid dimension and the numbers of nodes, robots, shelves, picking stations, products, their quantities, and orders. To guarantee reproducibility the header of each benchmark file gives the generator's version and instruction used to generate it.

The generator currently supports three types of layouts. A structured one, resembling real-world editions, a randomized one, and customized ones. In the structured layout, picking stations and robots are initially placed in the upper and lower row, respectively. Shelves are placed in rectangular clusters reachable via surrounding highways. An example is shown in Figure 1. No such structure is foreseen in a randomized layout. Finally, layouts can be handcrafted with asprilo's graphical editor (and afterwards populated with the instance generator). Moreover, the instance generator warrants that picking stations are not placed on highways and optionally that all admissible shelf positions are directly reachable from highway positions.

The instance generator is implemented via ASP. However, to handle the huge space of warehouse configurations and incoming orders, its implementation relies on multi-shot ASP solving and is controlled by the Python API of clingo (Gebser et al. 2018).

Although instances can be generated in a single solving step, benchmark sets are much more effectively generated incrementally by separating different sources of combinatorics. To this end, the generator relies upon an ASP encoding that is compartmentalized by means of clingo's \#program directive (cf. (Gebser et al. 2018)). Each such subprogram encapsulates a dimension of our scenario. Exemplary dimensions are the type of layout, the placement of shelves on admissible positions, and the one of products units on shelves (while respecting their global distribution). At first, the compartmentalization into subprograms allows us to configure the ultimate logic program according to the user's input. A typical example is the selection among layout alternatives. Moreover, the incremental approach takes advantage of compartmentalization by successively building benchmark 
instances in order to reduce combinatorics. At first, the grid is fixed, and robots and picking stations are placed. Alternatively, a handcrafted layout may be used (see below). Then, in turn, shelves, product units, and finally orders are generated. The overall process is controlled via clingo's Python API that successively grounds and solves the subprograms in focus. The incremental approach reduces combinatorics in two interesting ways. First, previous steps allow us to delineate the variability of objects in later steps. For instance, only admissible positions must be considered when placing shelves; similarly products must only be distributed among placed shelves. Second, we use configurable threshold-based domain splitting. To illustrate this, consider shelf placement and suppose we need to place 55 shelves on 100 positions. A splitting threshold of 20 makes the generator first choose 20 among 100 possible shelf positions. Then, the generator places 40 shelves on the 100 positions, but fixes the ones determined previously (by adding the corresponding facts); hence only 20 are effectively placed. And finally 55 shelves are placed, among which 40 are already fixed. Note that the selection of the respective threshold is a trade-off. Using the full set of objects is often infeasible, and although very small sets are easily treated, they produce many repetitive grounding and solving calls.

Finally, we mention that the generator features several ways of producing varied benchmark instances for the same configuration.

Now to accommodate the variety of its benchmark scenarios, asprilo's instance generator is highly configurable through a plethora of 68 options for specifying output instances, most notably the grid size, layout style, and object quantities. For example, the instance depicted in Fig. 1 is created with the command

$\begin{array}{lllllllllllllllllllllll}\text { gen } & -\mathrm{x} & 19 & -\mathrm{y} & 9 & -\mathrm{X} & 5 & -\mathrm{Y} & 2 & -\mathrm{p} & 3 & -\mathrm{s} & 45 & -\mathrm{r} & 6 & -\mathrm{P} & 180 & -\mathrm{u} & 540 & -\mathrm{o} & 12 & -\mathrm{H}\end{array}$

where parameter $-\mathrm{x}$ and $-\mathrm{y}$ fix the dimensions of the grid, $-\mathrm{X}$ and $-\mathrm{Y}$ the dimensions of the storage zones, $-p$ the number of picking stations, $-s$ the number of shelves, $-r$ the number of robots, $-\mathrm{P}$ the number of products, $-\mathrm{u}$ the number of product units, $-\mathrm{o}$ the number of orders, and $-\mathrm{H}$ invokes the structured layout common in industrial settings, involving highways and rectangular storage zones (along with the aforementioned systematic placement of robots and picking stations).

To produce more than one instance at a time, the desired number of instances can be given with -N. Further, option - I generates instances incrementally via multi-shot solving, as detailed above. For example, to incrementally generate 10 instances with our previous call, we just append $-\mathrm{N} 10$ and $-\mathrm{I}$ to the command line above.

Besides single invocations, asprilo also offers batch processing to generate multiple instance sets based on a list of configurations. ${ }^{8}$ Moreover, the batch file syntax not only allows us to process several configurations consecutively but also combinations thereof. That is, we can first stipulate a common (partial) preset of configuration parameters and afterwards derive more specialized sub-configurations from it by stating further parameter settings. For instance, we can initially pre-define the grid size, number of picking stations, shelves, products, and orders for all instances. Based on that preset, we can then define sub-configurations that state different amounts of robots. With it, the creation of complex instance sets can be fully defined (in a reproducible way) by a single batch file without the need for auxiliary scripts or manual curation.

8 The YAML vocabulary of batch files is described at http://potassco.org/asprilo. 


\subsection{Solution checker}

A solution to an asprilo scenario is a parallel plan represented by facts of the form occurs (object $(T, I)$, action $(A, V)$ ).

where $T$ is an object type, $I$ an object identifier, $A$ an action name, and $V$ a tuple of terms capturing the action's arguments. A move action takes cardinal points represented by $(0,1),(1,0),(0,-1)$, and $(-1,0)$. Actions pickup and putdown have no arguments, expressed by (). In general, deliver takes a triple $(O, A, N)$, where $O$ is an order, $A$ the delivered product, and $N$ its quantity. The latter is ignored in scenarios $\mathbf{B}$ and $\mathbf{C}$.

To verify the correctness of such plans, asprilo features a modular solution checker written in ASP. Depending on the scenario, the corresponding constraints are put together to form the appropriate checker. Apart from being modular, this offers an easily extensible approach for accommodating future extensions of asprilo.

Moreover, the checker's encoding offers diagnostic support indicating the respective violation. For this, it captures plan inconsistencies by returning atoms of the form $\operatorname{err}(F, C, P)$ where $F$ gives the name of the file defining the error, $C$ holds the name of the violated constraint, and $P$ specifies the parameters involved in its violation.

For example, suppose the checker returns the atom

err (goal, unfilledorder, $(3,3,1,11)$ )

This indicates that there is still an unfulfilled order at the end of the plan at step 11, namely, that order 3 still requires 1 unit of product 3. Further details are obtained by inspecting the rule in file goal.lp, which defines this specific instance of err $/ 3$.

\section{3 asprilo's visualization component}

The visualization component of asprilo serves two major purposes, the animation of solution candidates and the editing of benchmark templates. Such a template ranges from an empty scenario, over partial ones, to fully detailed warehouse environments. The latter can be accompanied by a (possibly invalid) parallel plan that can be animated in the supplied environment. Both are given in the previously described formats and can be passed to asprilo's visualizer either through files given on the command line, read from standard input, or loaded via a menu entry.

To begin with, let us sketch asprilo's animation capabilities. First of all, note that it is not coupled with the aforementioned solution checker. This is important to allow for debugging invalid solution candidates.

The states of an asprilo scenario are captured in a multi-modal way. First, the main window captures the position of objects as shown in Fig. 1. (In addition, robots carrying and being under shelves are indicated by distinct overlays of the respective icons.) Second, a side window gives a table reflecting the current status of all orders (reflecting closed and open order lines along with the delivered and missing product units). Finally, another side window lists the warehouse's inventory by displaying the product units from the shelves. The latter is also obtained when hovering with the mouse over the icon of a shelf in the main window. Similarly, information is obtainable on robots and picking stations.

State transitions are animated to simulate smooth robot movements. They can be traced via yet another side window holding the plan and highlighting all upcoming actions. The exploration of the plan at hand is done via common media controls (viz. 
play, pause, fast forward, etc.) to enact and change the animation. It comes without saying that the aforementioned state descriptions are updated accordingly.

Finally, we mention that scenarios and plans can be discharged and reloaded; also their textual representation can be edited.

The second major feature of asprilo's visualizer is its graphical scenario editor. With it, one may start from scratch with designing benchmark instances or templates, or modify existing designs. Templates can be further enriched by means of the above benchmark generator, leading to entire benchmark series. The functionality of asprilo's graphical editor allows for changing the grid dimensions, adding and removing robots, shelves, and stations, changing product units on shelves, declaring squares as highway or storage, and even entirely removing squares from the grid. The latter is of particular interest when designing non-uniform layouts, featuring obstacles such as corridors or pillars. Apart from the change of dimensions, all above functionalities are accomplished by mouse control. Also, objects can be moved by drag and drop. Similarly, orders can be edited textually in the corresponding side window.

A third feature, skipped for brevity, allows for connecting asprilo's visualizer with solving components over the network.

\section{Exemplary encodings}

We provide reference encodings for the various asprilo scenarios at https:// potassco.org/asprilo. For brevity, we illustrate here only our basic approach, viz. M, and merely sketch its extensions to more complex settings afterwards. Common to all our encodings is a mapping from asprilo's input format to a more comprehensible format. This is addressed with rules like ' $\operatorname{robot}(R)$ :- init (object (robot, $R$ ), _).' gathered in input. lp (cf. Line 1 in Listing 1). Another commonality of our encodings concerns the separation of the action theory from its goal specification, reflected by Listing 1 and 2 .

Listing 1 gives a bounded encoding for generating plans in asprilo scenario $\mathbf{M}$.

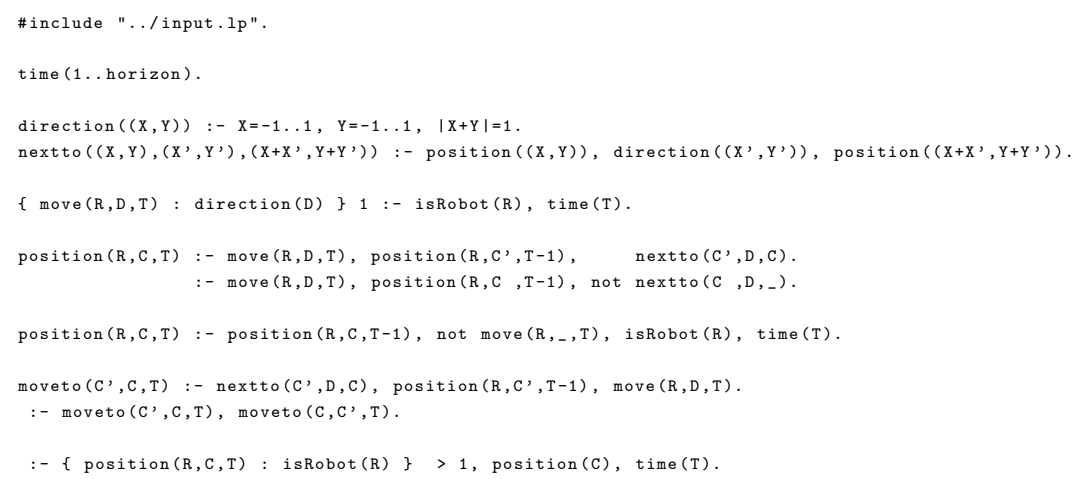

Listing 1. Encoding for asprilo domain $\mathbf{M}$.

The plan length is fixed by horizon in Line 3. Line 5 gives the four cardinal directions, used in Line 6 to represent all transitions on the warehouse grid. Predicate position/1 provides all grid coordinates, gathered in input.lp in analogy to the above. Line 8 generates all possible robot moves per time step. The corresponding effects and preconditions are expressed in Line 10 and 11, respectively. The inertia of robot positions, captured 
by position/3, is given in Line 13 . The remaining rules express state constraints. Lines 15 and 16 stipulate that robots must not swap positions, and Line 18 forbids more than one robot per square.

The drastically simplified concept of order fulfillment in $\mathbf{M}$ is encoded in Listing 2.

Listing 2. Encoding for order fulfillment in asprilo domain M.

An (ordered) product is processed according to Line 1, if some robot is parked at the last time step under a shelf holding the product. And the goal is fulfilled, if all ordered products have been processed.

We provide several alternative encodings for asprilo scenario $\mathbf{M}$, which mainly differ in how positions are represented. At first, we propose an encoding featuring a separate representation of coordinates. That is, rather than using predicate position $/ 3$ whose second argument is a pair (X,Y), we use two predicates positionX/3 and positionY/3 in which the second argument contains only one coordinate, namely $\mathrm{X}$ or $\mathrm{Y}$, respectively. This representation is similar to the one used for Constrained ASP (with clingcon (Banbara et al. 2017)), where both coordinates are represented functionally via integer variables positionX/2 and positionY/2 whose values give the $\mathrm{X}$ and $\mathrm{Y}$ coordinate, respectively. The same conceptualization is used for the fourth variant relying upon ASP enhanced with difference constraints (with clingo[DL] (Janhunen et al. 2017)) Also, we provide incremental versions of all but the last variant (since it is not multi-shot capable).

An even greater variety of encodings is made available for scenarios $\mathbf{A}, \mathbf{B}, \mathbf{C}$. Their common action theory deals with robot moves and pick up and put down actions. However, in addition to alternative representations of robots and shelves, we consider alternative representations of product units along with their combinations. The difference between $\mathbf{A}, \mathbf{B}$, and $\mathbf{C}$ is handled separately via different concepts of order fulfillment and thus different forms of delivery actions. Hence, as above, each setting boils down to two files, a specific encoding of the common action theory and another accounting for order fulfillment.

\section{Experimental evaluation}

To showcase asprilo's utility for experimenting with challenges emerging from robotic intra-logistics, we conducted some initial case studies using asprilo domains $\mathbf{M}$ and $\mathbf{C}^{\mathbf{M}}, \mathbf{B}^{\mathbf{M}}, \mathbf{A}^{\mathbf{M}}$ (in increasing difficulty). We give a detailed account of our experiments, including encodings, instance sets, and results at http://potassco.org/asprilo.

Our experimental analysis focuses on the following three questions:

1. What is the impact of different representations of grid positions?

2. What is the impact of increasingly complex domains?

3. What is the impact of decoupling sources of combinatorics?

We investigate the first question by comparing the four alternative encodings of positions discussed in Section 4. This is carried out in asprilo domain $\mathbf{M}$, since positions only change by movement. For the second question, we study the addition of actions and more complex goal conditions, and focus on domains $\mathbf{C}^{\mathbf{M}}, \mathbf{B}^{\mathbf{M}}, \mathbf{A}^{\mathbf{M}}$ as direct extensions 
of $\mathbf{M}$. The outcome of this question also reflects on the difference between traditionally studied, sparse multi-agent path finding (MAPF) scenarios (cf. Section 6) and richer warehouse scenarios as provided by asprilo. For the third question, we focus on separating task assignment and planning. To this end, we proceed in two steps. First, robots are assigned to transport certain shelves and to deliver at certain picking stations. This assignment is done subject to several optimization criteria that aim at selecting economic plans. Then, the resulting assignment is added to the original action theory along with constraints enforcing that actions are executed according to the given assignment. The use of explicit task assignments is related to the difference between anonymous and nonanonymous MAPF (cf. Section 6).

To minimize the degrees of freedom, we consider instances of $\mathbf{M}$ sharing a structured layout and storage zones fully covered by shelves. We divide the instance set into three groups, namely, small, medium, and large, according to their increasing complexity. To be more precise, their layout sizes are $11 \times 6,19 \times 9,46 \times 15$ with $16,60,320$ storage locations, respectively. ${ }^{9}$ Besides layout size, we categorize instances by number of robots:

- for small instances: increments of $2,5,8$ and 11 robots,

- for medium instances: increments of 5, 10, 15 and 19 robots, and

- for large instances: increments of $12,23,35$ and 46 robots.

We consider 30 instances per robot increment, and hence 120 per size and 360 in total.

Our experiments ran under Linux on an AMD Opteron 6278 with 32 cores and 256 GB, using clingo 5.3 and clingcon 3.3 in their standard configurations. Each run was limited to one thread on one physical core, 1800s, and 8 GB. Notably, each instance is bound by its smallest pre-calculated makespan, which makes the instances particularly hard to solve. This makespan is calculated by the incremental encodings mentioned in Section 4 subject to a timeout of $8 \mathrm{~h}$. Whenever the smallest makespan cannot be determined within this time limit, we also consider the instance as unsolvable in the domain at hand. Also, assignments are pre-computed and the same assignment is used for all encodings in the same domain. The computation of feasible assignments is more or less instantaneous; their optimization was cut after 300s (but this only happens with large instances).

Our results are summarized in Table 1. The first column gives the respective asprilo domain; experiments with prior task assignments are indicated by adding subscript a. The second column gives the average makespan of each size. For instance, for $\mathbf{M}_{\mathbf{a}}$ the average makespans are 6, 10, and 25 for small, medium and large instances; '-' tells us that the pre-computation of the makespan timed out for all instances in the domain. The third column reflects the respective approach by giving the target system of the encoding. As detailed in Section 4, clingo stands for the encoding in Listing 1 and 2 (and its extensions), clingo $_{x y}$ for the ASP encoding splitting coordinates, clingo[DL] for the encoding mapping coordinates on two integer variables subject to difference constraints, and analogously clingcon but using linear constraints. Notably, clingcon's constraint processing is extended in $\mathbf{A}$ to product units. The last three columns give average run time in seconds followed by the number of timeouts in parenthesis obtained for task

9 For the reader's convenience, we give the corresponding generator calls and layouts in Table A1. 
Table 1. Summary of experimental results in average run time and number of timeouts.

\begin{tabular}{|c|c|c|c|c|c|}
\hline domain & makespan & encoding & small & medium & large \\
\hline M & $6 / 10 / 25$ & $\begin{array}{c}\text { clingo } \\
\text { clingo }_{x y} \\
\text { clingcon } \\
\text { clingo }[\mathrm{DL}]\end{array}$ & $\begin{array}{l}0(0) \\
0(0) \\
0(0) \\
0(0)\end{array}$ & $\begin{array}{r}\mathbf{0 ( 0 )} \\
16(1) \\
37(0) \\
193(1)\end{array}$ & $\begin{array}{r}\mathbf{7 3 ( 4 )} \\
591(14) \\
1168(52) \\
1648(96)\end{array}$ \\
\hline $\mathrm{M}_{\mathrm{a}}$ & $6 / 10 / 25$ & $\begin{array}{c}\text { clingo } \\
\text { clingo }_{x y} \\
\text { clingcon } \\
\text { clingo }[\mathrm{DL}]\end{array}$ & $\begin{array}{l}0(0) \\
0(0) \\
0(0) \\
0(0)\end{array}$ & $\begin{array}{r}\mathbf{0 ( 0 )} \\
\mathbf{0 ( 0 )} \\
36(0) \\
86(1)\end{array}$ & $\begin{array}{r}\mathbf{4 1}(\mathbf{2}) \\
763(27) \\
1163(49) \\
1679(102)\end{array}$ \\
\hline $\mathrm{C}^{\mathrm{M}}$ & $20 /-/-$ & $\begin{array}{c}\text { clingo } \\
\text { clingcon }\end{array}$ & $\begin{array}{l}805(40) \\
\mathbf{6 9 5}(\mathbf{3 0})\end{array}$ & $\begin{array}{l}1800(120) \\
1800(120)\end{array}$ & $\begin{array}{l}1800(120) \\
1800(120)\end{array}$ \\
\hline $\mathrm{C}_{\mathrm{a}}^{\mathrm{M}}$ & $21 / 35 /-$ & $\begin{array}{l}\text { clingo } \\
\text { clingcon }\end{array}$ & $\begin{array}{l}\mathbf{2 3}(\mathbf{1}) \\
38(2)\end{array}$ & $\begin{array}{r}\mathbf{3 7 0}(\mathbf{5}) \\
459(15)\end{array}$ & $\begin{array}{l}1800(120) \\
1800(120)\end{array}$ \\
\hline $\mathbf{B}^{\mathrm{M}}$ & $26 /-/-$ & $\begin{array}{c}\text { clingo } \\
\text { clingcon }\end{array}$ & $\begin{array}{l}970(53) \\
\mathbf{8 0 7}(\mathbf{3 7})\end{array}$ & $\begin{array}{l}1800(120) \\
1800(120)\end{array}$ & $\begin{array}{l}1800(120) \\
1800(120)\end{array}$ \\
\hline $\mathbf{B}_{\mathrm{a}}^{\mathrm{M}}$ & 26/39/- & $\begin{array}{c}\text { clingo } \\
\text { clingcon }\end{array}$ & $\begin{array}{l}\mathbf{1 2}(\mathbf{0}) \\
29(0)\end{array}$ & $\begin{array}{l}\mathbf{5 6 6}(\mathbf{1 9}) \\
623(25)\end{array}$ & $\begin{array}{l}1800(120) \\
1800(120)\end{array}$ \\
\hline $\mathbf{A}^{\mathrm{M}}$ & $26 /-/-$ & $\begin{array}{c}\text { clingo } \\
\text { clingcon }\end{array}$ & $\begin{array}{l}984(55) \\
\mathbf{8 5 6 ( 4 1 )}\end{array}$ & $\begin{array}{l}1800(120) \\
1800(120)\end{array}$ & $\begin{array}{l}1800(120) \\
1800(120)\end{array}$ \\
\hline $\mathbf{A}_{\mathrm{a}}^{\mathrm{M}}$ & $26 / 39 /-$ & $\begin{array}{l}\text { clingo } \\
\text { clingcon }\end{array}$ & $\begin{array}{l}\mathbf{1 2 ( 0 )} \\
49(1)\end{array}$ & $\begin{array}{l}\mathbf{5 7 7}(\mathbf{1 8}) \\
625(22)\end{array}$ & $\begin{array}{l}1800(120) \\
1800(120)\end{array}$ \\
\hline
\end{tabular}

planning; the best one in terms of timeouts (and secondarily time) is bold faced. Timeouts account for 1800s; run-times less than 5s are marked as $0 .{ }^{10}$

All encodings, except for the one related to clingo, split positional coordinates. While this is necessary with clingcon and clingo[DL] due to their use of integer variables, clingo $_{x y}$ allows us to examine the effect of splitting in plain ASP. Contrary to our expectations, this technique hardly led to performance improvements in our experiments. Specifically, for $\mathbf{M}$ and $\mathbf{M}_{\mathbf{a}}$, the clingo encoding beats all others by a notable margin. Although the other encodings produce smaller ground programs in these domains, in case of clingcon and clingo[DL] even by an order of magnitude, the resulting number of constraints seems to increase. ${ }^{11}$ This changes for $\mathbf{A}^{\mathbf{M}}, \mathbf{B}^{\mathbf{M}}$, and $\mathbf{C}^{\mathbf{M}}$, where clingcon takes the lead; and roles switch again when prior task assignments are used as in $\mathbf{A}_{\mathbf{a}}^{\mathbf{M}}, \mathbf{B}_{\mathbf{a}}^{\mathbf{M}}$, and $\mathbf{C}_{\mathbf{a}}^{\mathbf{M}}$. We conjecture that this change is due to the reduction in problem size caused by the added task assignment (see below), and that clingcon's solving techniques gain more effect on larger instances. However, the catch-up effect of clingcon in this context cannot clearly be traced back to coordinate splitting only. Interestingly, the small integer domains disable clingcon's lazy propagation, so that all constraints are compiled out by using the order encoding (Tamura et al. 2009). In fact, this offers a higher propagation strength than the direct encoding used in the clingo encoding.

10 For the reader's convenience, we give more fine-grained results in Table B1, B2 and B3 in the appendix. As mentioned, the full details of our experiments are given at http://potassco.org/asprilo.

11 This data was not available for clingo[DL]. 
In the simplistic $\mathbf{M}$ domain, all instance sizes are almost completely solvable. Timeouts only occur for larger instances with 23 or more robots. The transition to $\mathbf{C}^{\mathbf{M}}$ severely increases the average minimal makespan and therefore the run time. In particular, only small instances for robot increments 2, 5 and 8 are solvable in time for the most part, whereas all medium and large instances time out. This limitation carries over to $\mathbf{B}^{\mathbf{M}}$ and $\mathbf{A}^{\mathbf{M}}$. The more time-consuming delivery action in $\mathbf{B}^{\mathbf{M}}$ (and $\mathbf{A}^{\mathbf{M}}$ ) further increases the average minimal makespan in comparison to $\mathbf{C}^{\mathbf{M}}$. Conversely, the addition of product quantities by $\mathbf{A}^{\mathbf{M}}$ has no further impact on the average plan lengths. Likewise, there is a notable linear increase of run times between $\mathbf{C}^{\mathbf{M}}$ and $\mathbf{B}^{\mathbf{M}}$ whereas the difference between $\mathbf{B}^{\mathbf{M}}$ and $\mathbf{A}^{\mathbf{M}}$ is relatively small. This to be expected since only singleton product units are considered in $\mathbf{A}^{\mathbf{M}}{ }^{12}$ So, units are taken into account but not extensively manipulated.

Our analysis has already made it apparent that a "one-encoding-fits-all" approach fails to scale on industrial size instances. On the other hand, the design of the instance generator has revealed how the compartmentalization of sources of combinatorics allows for effectively reducing the overall difficulty of a problem. This is confirmed by our experiments on the decoupling of task assignment and planning. The provision of assignments improved scalability in each domain. Especially for $\mathbf{C}_{\mathbf{a}}^{\mathbf{M}}, \mathbf{B}_{\mathbf{a}}^{\mathbf{M}}$, and $\mathbf{A}_{\mathbf{a}}^{\mathbf{M}}$, the average run times decrease by at least an order of magnitude and almost all small and medium instances up to robot increment 15 become solvable within the time limit. ${ }^{13}$ Let us detail this by examining the path finding in instance x11_y6_n66_r8_s16_ps1_pr16_u16_o8_N001.1p in domain $\mathbf{A}^{\mathbf{M}}$ with and without decoupling. Although the added assignment slightly increases the incoming program size from 774584 rules and 65294 atoms to 818321 rules and 68284 atoms, the internal representation obtained after preprocessing is much smaller, namely, 1400191 constraints over 295835 atoms versus 389874 constraints over 102316 atoms. That is, here, the assignment makes the number of constraints shrink by an order of magnitude and halves the number of variables. This reduction is less pronounced with clingcon, where the number of rules/atoms and constraints/atoms drops from 143137/70127, $677131 / 95934$ to $130064 / 59957,292149 / 70666$. Likewise, the difficulty of search in clingo is decreased by several orders of magnitude from 10807510 choices and 562464 conflicts to 107732 choices and 28330 conflicts. Finally, our efforts to obtain economic plans pay off, and the pre-determined task assignments lead to no increase of the smallest makespans, except for $\mathbf{C}^{\mathbf{M}}$ where it increases from 20 to 21 . Obviously, the makespans' stability is due to the optimized task assignments. However, the problem reduction as such is obtained with any assignment. Considering the large performance gain, optimized assignments constitute an interesting trade-off for solution quality. Moreover, this trade-off is easy to control since optimization can be done in an anytime manner.

\section{Related work}

Our motivation is very similar to the early work on TheoryBase (Cholewiński et al. 1995), which provided our community with a systematic way of creating logic programs from graph-based combinatorial problems. Recently, for instance, more application-oriented combinatorial problems were proposed in (Abseher et al. 2016; Banbara et al. 2016;

12 Increasing product units need intensive experimentation and are thus left to future research.

13 We have no explanation why encoding clingo $_{x y}$ deteriorates when adding task assignments. 
Alviano et al. 2017), dealing with shift planning, course timetabling, and nurse scheduling, respectively. Unlike these static problems, the decision support system for the space shuttle (Nogueira et al. 2001) and the Ricochet Robots board game (Gebser et al. 2013) were both put forward as dynamic benchmark problems, also involving multiple, collaboratively controlled robots. And many more can be found in the repositories of last years' ASP competitions. What distinguishes robotic intra-logistics is its multidimensional nature that necessitates the integration of a great many of aspects.

At the core of many path finding problems lies the search for a route for an agent from an initial to a final location. The multi-agent path finding (MAPF) problem asks for a collision-free route for each agent such that the total makespan is minimal. MAPF is related to many real-world applications but already computationally intractable (Surynek 2010). While in MAPF each agent is assigned a unique destination, its anonymous variant requires no assignment of agents to destinations ( $\mathrm{Yu}$ and LaValle 2012). The problem domains of asprilo are obviously related to multi-agent path finding. More specifically, the asprilo domain $\mathbf{M}$ corresponds to anonymous MAPF. Each order is uniquely associated with a destination shelf and there is no pre-assignment of a robot to an order. Robots can freely reach any destination shelf. Clearly, $\mathbf{M}$ is easily extended to cover non-anonymous MAPF by relating robots and orders.

Task assignment and path finding (TAPF; Ma and Koenig 2016) is a generalization of MAPF. TAPF groups agents into teams. Although teams are (non-anonymously) preassigned to groups of destinations, any robot in the team can be (anonymously) selected for a destination in the assigned group. G-TAPF (Nguyen et al. 2017) is a generalization of TAPF aiming at more realistic settings by allowing the number of tasks to be greater than the number of agents and considering deadlines, orderings, and checkpoints. That is, deadlines are associated with order lines, orders are completed in a pre-defined ordering and all lines in a single order need to be fulfilled before any line of another order is completed, and while fulfilling an order, a robot is required to go through a sequence of locations, called checkpoints. Note that asprilo's generic instance format can be easily extended to handle key concepts of TAPF and G-TAPF (like robot teams, deadlines, or checkpoints) and we plan to offer corresponding scenarios in the near future. Regarding previous uses of ASP, (Erdem et al. 2013) address several aspects of multi-agent path finding problems. The asprilo framework is obviously beneficial for boosting such work.

Ma et al. (2017) address an online version of path finding, where not all destination tasks $^{14}$ are given initially but may arrive over time. In view of the availability of multi-shot ASP solving, the treatment of online versions of asprilo's problem domains constitutes an important future line of research for us. Several emerging requirements of online intralogistic problems have already been taken into account in the design of asprilo.

\section{Conclusion}

The current asprilo system provides a basic infrastructure for experimental studies of dynamic systems. As is, it already presents a number of challenges to ASP-based approaches for solving intra-logistics problems, whenever scalability and efficiency are at

14 Actually, this work also uses pick-up and delivery tasks to simulate a warehouse system. 
stake. Abstraction and distribution appear to be promising avenues of future research towards scalability. Thanks to its open design, it can be extended in various ways. Dealing with preferences, different types of orders, and uncertainty provide additional significant challenges that might require new insights or approaches. Last but not least, online approaches to solving intra-logistics problems are interesting and might even be necessary.

To sum up, we have presented asprilo, a framework for experimental studies of dynamic systems, specifically in the intra-logistics domains. We discussed our design decisions and detailed the different components of asprilo: its benchmark generator, solution checker, visualizer, and sample encodings. We illustrated asprilo's utility of providing an experimental platform for solving various problems in robotic intra-logistics and revealed significant challenges to our community in closing the gap to industrial-scale applications.

Videos illustrating asprilo in domains $\mathbf{A}$ and $\mathbf{M}$ can be found https://youtu.be/ ifYKHIvdnjw and https://youtu.be/GHRwpWzL0j8, respectively.

Acknowledgments. This work was partially supported by The Scientific and Technological Research Council of Turkey (TUBITAK) under project 117C044 and DFG grant SCHA $550 / 9$.

\section{References}

Abseher, M., Gebser, M., Musliu, N., Schaub, T., and Woltran, S. 2016. Shift design with answer set programming. Fundamenta Informaticae 147, 1, 1-25.

Alviano, M., Dodaro, C., And Maratea, M. 2017. An advanced answer set programming encoding for nurse scheduling. In Proceedings of the Sixteenth International Conference of the Italian Association for Artificial Intelligence, F. Esposito, R. Basili, S. Ferilli, and F. Lisi, Eds. Lecture Notes in Computer Science, vol. 10640. Springer-Verlag, 468-482.

Banbara, M., Inoue, K., Kaufmann, B., Schaub, T., Soh, T., Tamura, N., and Wanko, P. 2016. teaspoon: Solving the curriculum-based course timetabling problems with answer set programming. In Proceedings of the Eleventh International Conference of the Practice and Theory of Automated Timetabling (PATAT'16), E. Burke, L. D. Gaspero, B. McCollum, A. Schaerf, and E. Özcan, Eds. 13-32.

Banbara, M., Kaufmann, B., Ostrowski, M., And Schaub, T. 2017. Clingcon: The next generation. Theory and Practice of Logic Programming 17, 4, 408-461.

Cholewiński, P., Marek, V., Mikitiuk, A., And Truszczyński, M. 1995. Experimenting with nonmonotonic reasoning. In Proceedings of the International Conference on Logic Programming, L. Sterling, Ed. MIT Press, 267-281.

Erdem, E., KisA, D., Öztok, U., ANd Schüller, P. 2013. A general formal framework for pathfinding problems with multiple agents. In Proceedings of the Twenty-Seventh National Conference on Artificial Intelligence (AAAI'13), M. desJardins and M. Littman, Eds. AAAI Press, 290-296.

Gebser, M., Jost, H., Kaminski, R., Obermeier, P., Sabuncu, O., Schaub, T., And Schneider, M. 2013. Ricochet robots: A transverse ASP benchmark. In Proceedings of the Twelfth International Conference on Logic Programming and Nonmonotonic Reasoning (LPNMR'13), P. Cabalar and T. Son, Eds. Lecture Notes in Artificial Intelligence, vol. 8148. Springer-Verlag, 348-360.

Gebser, M., Kaminski, R., Kaufmann, B., and Schaub, T. 2018. Multi-shot ASP solving with clingo. Theory and Practice of Logic Programming. To appear.

Janhunen, T., Kaminski, R., Ostrowski, M., Schaub, T., Schellhorn, S., And Wanko, P. 2017. Clingo goes linear constraints over reals and integers. Theory and Practice of Logic Programming 17, 5-6, 872-888. 
Lifschitz, V. 1999. Answer set planning. In Proceedings of the International Conference on Logic Programming (ICLP'99), D. de Schreye, Ed. MIT Press, 23-37.

Ma, H. And Koenig, S. 2016. Optimal target assignment and path finding for teams of agents. In Proceedings of the Fifteenth International Conference on Autonomous Agents and Multiagent Systems (AAMAS'16), C. Jonker, S. Marsella, J. Thangarajah, and K. Tuyls, Eds. ACM Press, 1144-1152.

Ma, H., Li, J., Kumar, T., And Koenig, S. 2017. Lifelong multi-agent path finding for online pickup and delivery tasks. In Proceedings of the Sixteenth Conference on Autonomous Agents and MultiAgent Systems (AAMAS'17). ACM Press, 837-845.

Nguyen, V., Obermeier, P., Son, T., Schaub, T., and Yeoh, W. 2017. Generalized target assignment and path finding using answer set programming. In Proceedings of the Twenty-sixth International Joint Conference on Artificial Intelligence (IJCAI'17), C. Sierra, Ed. IJCAI/AAAI Press, 1216-1223.

Nogueira, M., Balduccini, M., Gelfond, M., Watson, R., And Barry, M. 2001. An Aprolog decision support system for the space shuttle. In Proceedings of the Third International Symposium on Practical Aspects of Declarative Languages (PADL'01), I. Ramakrishnan, Ed. Lecture Notes in Computer Science, vol. 1990. Springer-Verlag, 169-183.

SuRYNEK, P. 2010. An optimization variant of multi-robot path planning is intractable. In Proceedings of the Twenty-fourth National Conference on Artificial Intelligence (AAAI'10), M. Fox and D. Poole, Eds. AAAI Press, 1261-1263.

Tamura, N., Taga, A., Kitagawa, S., and Banbara, M. 2009. Compiling finite linear CSP into SAT. Constraints 14, 2, 254-272.

Yu, J. ANd LaValle, S. 2012. Multi-agent path planning and network flow. In Proceedings of the Tenth Workshop on the Algorithmic Foundations of Robotics (WAFR'12), E. Frazzoli, T. Lozano-Pérez, N. Roy, and D. Rus, Eds. Springer Tracts in Advanced Robotics, vol. 86. Springer-Verlag, 157-173. 


\section{Appendix A. Benchmark instance layout}

Table A1. Instance layout sizes specified by generator calls and resulting layouts; particularly, the depicted calls show the first robot increment per layout size with the related parameters for robots and orders colored in blue.

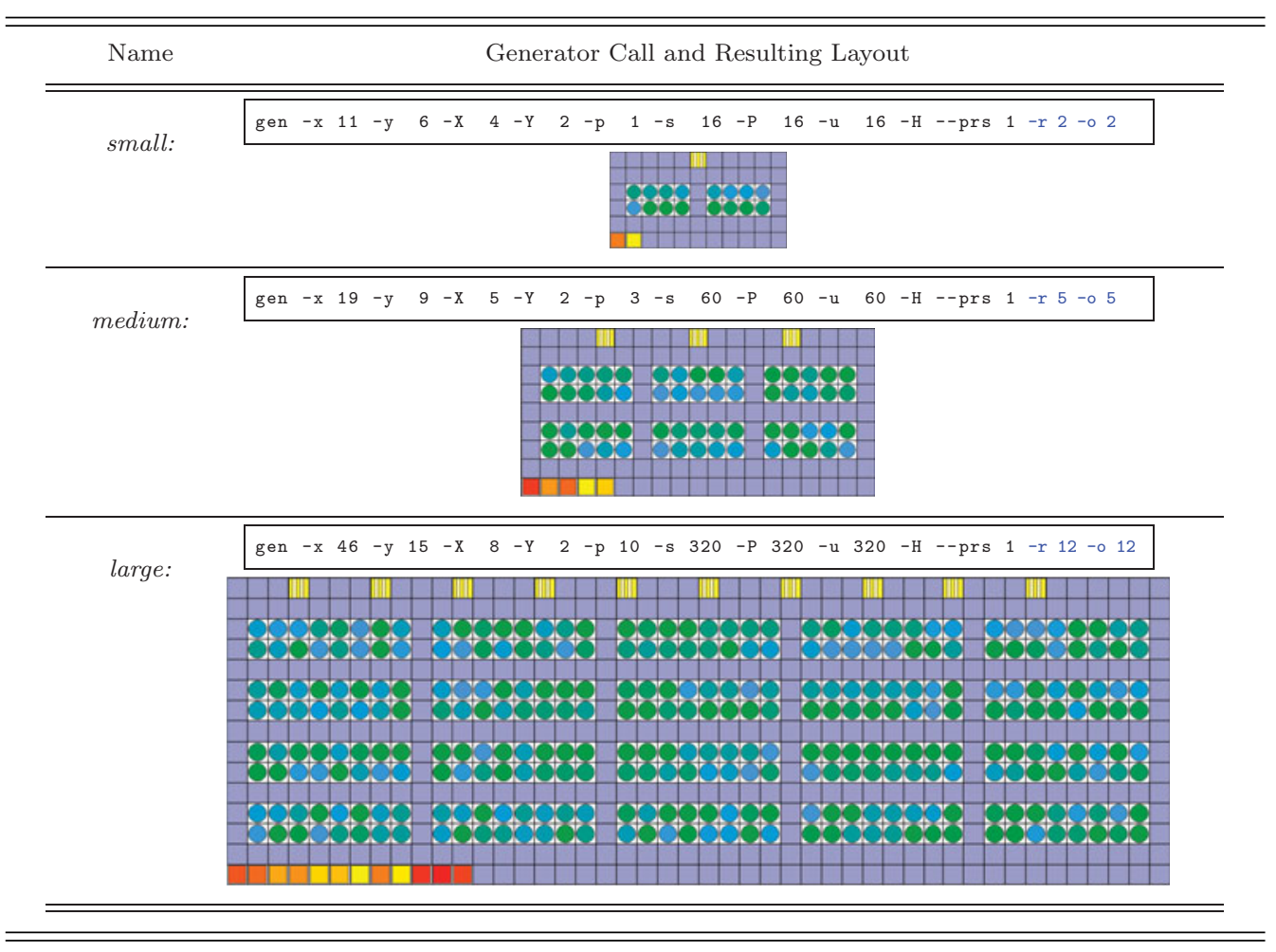




\section{Appendix B. Detailed evaluation results}

Table B1. Average run time and number of time outs for small instances.

\begin{tabular}{|c|c|c|c|c|c|c|}
\hline domain & makespan & encoding & 2 robots & 5 robots & 8 robots & 11 robots \\
\hline \multirow{4}{*}{$\mathbf{M}$} & \multirow{4}{*}{6} & clingo & $0(0)$ & $0(0)$ & $0(0)$ & $0(0)$ \\
\hline & & $\operatorname{clingo}_{x y}$ & $0(0)$ & $0(0)$ & $0(0)$ & $0(0)$ \\
\hline & & clingcon & $0(0)$ & $0(0)$ & $0(0)$ & $0(0)$ \\
\hline & & clingo $[\mathrm{DL}]$ & $0(0)$ & $0(0)$ & $0(0)$ & $0(0)$ \\
\hline \multirow{4}{*}{$\mathrm{M}_{\mathrm{a}}$} & \multirow{4}{*}{6} & clingo & $0(0)$ & $0(0)$ & $0(0)$ & $0(0)$ \\
\hline & & clingo $_{x y}$ & $0(0)$ & $0(0)$ & $0(0)$ & $0(0)$ \\
\hline & & clingcon & $0(0)$ & $0(0)$ & $0(0)$ & $0(0)$ \\
\hline & & clingo $[\mathrm{DL}]$ & $0(0)$ & $0(0)$ & $0(0)$ & $0(0)$ \\
\hline \multirow{2}{*}{$\mathrm{C}^{\mathrm{M}}$} & \multirow{2}{*}{20} & clingo & $46(0)$ & $751(9)$ & $623(1)$ & $1800(30)$ \\
\hline & & clingcon & $17(0)$ & $580(5)$ & $411(0)$ & $1772(25)$ \\
\hline \multirow{2}{*}{$\mathrm{C}_{\mathrm{a}}^{\mathrm{M}}$} & \multirow{2}{*}{21} & clingo & $0(0)$ & $4(0)$ & $67(1)$ & $21(0)$ \\
\hline & & clingcon & $61(1)$ & $4(0)$ & $9(0)$ & $80(1)$ \\
\hline \multirow{2}{*}{$\mathbf{B}^{\mathrm{M}}$} & \multirow{2}{*}{26} & clingo & $37(0)$ & $398(1)$ & $1647(22)$ & $1800(30)$ \\
\hline & & clingcon & $15(0)$ & $254(0)$ & $1159(7)$ & $1800(30)$ \\
\hline \multirow{2}{*}{$\mathbf{B}_{\mathrm{a}}^{\mathrm{M}}$} & \multirow{2}{*}{26} & clingo & $0(0)$ & $5(0)$ & $23(0)$ & $22(0)$ \\
\hline & & clingcon & $0(0)$ & $6(0)$ & $15(0)$ & $94(0)$ \\
\hline \multirow{2}{*}{$\mathbf{A}^{\mathbf{M}}$} & \multirow{2}{*}{26} & clingo & $52(0)$ & $422(2)$ & $1662(23)$ & $1800(30)$ \\
\hline & & clingcon & $18(0)$ & $303(1)$ & $1304(10)$ & $1800(30)$ \\
\hline \multirow{2}{*}{$\mathbf{A}_{\mathrm{a}}^{\mathrm{M}}$} & \multirow{2}{*}{26} & clingo & $0(0)$ & $0(0)$ & $25(0)$ & $20(0)$ \\
\hline & & clingcon & $0(0)$ & $0(0)$ & $16(0)$ & $175(1)$ \\
\hline
\end{tabular}


Table B2. Average run time and number of timeouts for medium-sized instances.

\begin{tabular}{|c|c|c|c|c|c|c|}
\hline domain & makespan & encoding & 5 robots & 10 robots & 15 robots & 19 robots \\
\hline \multirow{4}{*}{ M } & \multirow{4}{*}{10} & clingo & $0(0)$ & $0(0)$ & $0(0)$ & $0(0)$ \\
\hline & & clingo $_{x y}$ & $0(0)$ & $0(0)$ & $61(1)$ & $3(0)$ \\
\hline & & clingcon & $0(0)$ & $0(0)$ & $11(0)$ & $135(0)$ \\
\hline & & clingo $[\mathrm{DL}]$ & $5(0)$ & $120(1)$ & $180(0)$ & $467(0)$ \\
\hline \multirow{4}{*}{$\mathrm{M}_{\mathrm{a}}$} & \multirow{4}{*}{10} & clingo & $0(0)$ & $0(0)$ & $0(0)$ & $0(0)$ \\
\hline & & clingo $_{x y}$ & $0(0)$ & $0(0)$ & $0(0)$ & $0(0)$ \\
\hline & & clingcon & $0(0)$ & $0(0)$ & $16(0)$ & $128(0)$ \\
\hline & & clingo $[\mathrm{DL}]$ & $18(0)$ & $150(1)$ & $76(0)$ & $103(0)$ \\
\hline \multirow{2}{*}{$\mathrm{C}^{\mathrm{M}}$} & \multirow{2}{*}{ - } & clingo & $1800(30)$ & $1800(30)$ & $1800(30)$ & $1800(30)$ \\
\hline & & clingcon & $1800(30)$ & $1800(30)$ & $1800(30)$ & $1800(30)$ \\
\hline \multirow{2}{*}{$\mathrm{C}_{\mathrm{a}}^{\mathrm{M}}$} & \multirow{2}{*}{35} & clingo & $50(0)$ & $106(0)$ & $310(0)$ & $1015(5)$ \\
\hline & & clingcon & $41(0)$ & $116(0)$ & $512(2)$ & $1169(13)$ \\
\hline \multirow{2}{*}{$\mathbf{B}^{\mathrm{M}}$} & \multirow{2}{*}{ - } & clingo & $1800(30)$ & $1800(30)$ & $1800(30)$ & $1800(30)$ \\
\hline & & clingcon & $1800(30)$ & $1800(30)$ & $1800(30)$ & $1800(30)$ \\
\hline \multirow{2}{*}{$\mathbf{B}_{\mathrm{a}}^{\mathrm{M}}$} & \multirow{2}{*}{39} & clingo & $53(0)$ & $151(0)$ & $569(1)$ & $1492(18)$ \\
\hline & & clingcon & $47(0)$ & $159(0)$ & $709(3)$ & $1579(22)$ \\
\hline \multirow{2}{*}{$\mathrm{A}^{\mathrm{M}}$} & \multirow{2}{*}{ - } & clingo & $1800(30)$ & $1800(30)$ & $1800(30)$ & $1800(30)$ \\
\hline & & clingcon & $1800(30)$ & $1800(30)$ & $1800(30)$ & $1800(30)$ \\
\hline \multirow{2}{*}{$\mathbf{A}_{\mathrm{a}}^{\mathrm{M}}$} & \multirow{2}{*}{39} & clingo & $59(0)$ & $159(0)$ & $594(1)$ & $1497(17)$ \\
\hline & & clingcon & $48(0)$ & $143(0)$ & 701(0) & $1608(22)$ \\
\hline
\end{tabular}

Table B3. Average run time and number of timeouts for large instances.

\begin{tabular}{|c|c|c|c|c|c|c|}
\hline domain & makespan & encoding & 12 robots & 23 robots & 35 robots & 46 robots \\
\hline M & 25 & $\begin{array}{c}\text { clingo } \\
\text { clingo } x y \\
\text { clingcon } \\
\text { clingo }[\mathrm{DL}]\end{array}$ & $\begin{array}{r}\mathbf{1 5 ( 0 )} \\
205(0) \\
119(0) \\
1193(6)\end{array}$ & $\begin{array}{r}\mathbf{7 9}(\mathbf{1}) \\
1007(7) \\
1108(3) \\
1800(30)\end{array}$ & $\begin{array}{r}\mathbf{1 5 ( 0 )} \\
808(4) \\
1748(27) \\
1800(30)\end{array}$ & $\begin{array}{r}\mathbf{1 8 6}(\mathbf{3}) \\
347(3) \\
1700(22) \\
1800(30)\end{array}$ \\
\hline $\mathrm{M}_{\mathrm{a}}$ & 25 & $\begin{array}{c}\text { clingo } \\
\text { clingo }_{x y} \\
\text { clingcon } \\
\text { clingo }[\mathrm{DL}]\end{array}$ & $\begin{array}{r}\mathbf{1 3}(\mathbf{0}) \\
249(1) \\
162(1) \\
1316(12)\end{array}$ & $\begin{array}{r}\mathbf{1 8}(\mathbf{0}) \\
1282(15) \\
1053(0) \\
1800(30)\end{array}$ & $\begin{array}{r}\mathbf{1 0}(\mathbf{0}) \\
1096(7) \\
1734(25) \\
1800(30)\end{array}$ & $\begin{array}{r}\mathbf{1 2 5}(\mathbf{2}) \\
427(4) \\
1706(23) \\
1800(30)\end{array}$ \\
\hline $\mathrm{C}^{\mathrm{M}}$ & - & $\begin{array}{c}\text { clingo } \\
\text { clingcon }\end{array}$ & $\begin{array}{l}1800(30) \\
1800(30)\end{array}$ & $\begin{array}{l}1800(30) \\
1800(30)\end{array}$ & $\begin{array}{l}1800(30) \\
1800(30)\end{array}$ & $\begin{array}{l}1800(30) \\
1800(30)\end{array}$ \\
\hline $\mathrm{C}_{\mathbf{a}}^{\mathrm{M}}$ & - & $\begin{array}{l}\text { clingo } \\
\text { clingcon }\end{array}$ & $\begin{array}{l}1800(30) \\
1800(30)\end{array}$ & $\begin{array}{l}1800(30) \\
1800(30)\end{array}$ & $\begin{array}{l}1800(30) \\
1800(30)\end{array}$ & $\begin{array}{l}1800(30) \\
1800(30)\end{array}$ \\
\hline $\mathbf{B}^{\mathrm{M}}$ & - & $\begin{array}{l}\text { clingo } \\
\text { clingcon }\end{array}$ & $\begin{array}{l}1800(30) \\
1800(30)\end{array}$ & $\begin{array}{l}1800(30) \\
1800(30)\end{array}$ & $\begin{array}{l}1800(30) \\
1800(30)\end{array}$ & $\begin{array}{l}1800(30) \\
1800(30)\end{array}$ \\
\hline $\mathbf{B}_{\mathbf{a}}^{\mathrm{M}}$ & - & $\begin{array}{l}\text { clingo } \\
\text { clingcon }\end{array}$ & $\begin{array}{l}1800(30) \\
1800(30)\end{array}$ & $\begin{array}{l}1800(30) \\
1800(30)\end{array}$ & $\begin{array}{l}1800(30) \\
1800(30)\end{array}$ & $\begin{array}{l}1800(30) \\
1800(30)\end{array}$ \\
\hline $\mathbf{A}^{\mathrm{M}}$ & - & $\begin{array}{c}\text { clingo } \\
\text { clingcon }\end{array}$ & $\begin{array}{l}1800(30) \\
1800(30)\end{array}$ & $\begin{array}{l}1800(30) \\
1800(30)\end{array}$ & $\begin{array}{l}1800(30) \\
1800(30)\end{array}$ & $\begin{array}{l}1800(30) \\
1800(30)\end{array}$ \\
\hline $\mathbf{A}_{\mathrm{a}}^{\mathrm{M}}$ & - & $\begin{array}{l}\text { clingo } \\
\text { clingcon }\end{array}$ & $\begin{array}{l}1800(30) \\
1800(30)\end{array}$ & $\begin{array}{l}1800(30) \\
1800(30)\end{array}$ & $\begin{array}{l}1800(30) \\
1800(30)\end{array}$ & $\begin{array}{l}1800(30) \\
1800(30)\end{array}$ \\
\hline
\end{tabular}

